\title{
The Formulation and Justification of Mathematical Definitions Illustrated By Deterministic Chaos
}

\author{
Charlotte Werndl \\ Faculty of Philosophy, University of Cambridge, Sidgwick Avenue, Cambridge, CB3 9DA, \\ $U K$
}

\begin{abstract}
The general theme of this article is the actual practice of how definitions are justified and formulated in mathematics. The theoretical insights of this article are based on a case study of topological definitions of chaos. After introducing this case study, I identify the three kinds of justification which are important for topological definitions of chaos: natural-world-justification, condition-justification and redundancy-justification. To my knowledge, the latter two have not been identified before. I argue that these three kinds of justification are widespread in mathematics. After that, I first discuss the state of the art in the literature about the justification of definitions in the light of actual mathematical practice. I then go on to criticize Lakatos's account of proofgenerated definitions - the main account in the literature on this issue - as being limited and also misguided: as for topological definitions of chaos, in nearly all mathematical fields various kinds of justification are found and are also reasonable.
\end{abstract}

Table of Contents

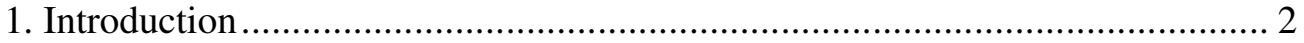

2. Case Study: Topological Definitions of Chaos ............................................ 3

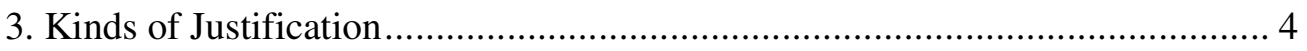

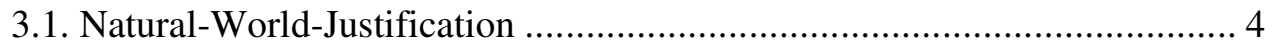

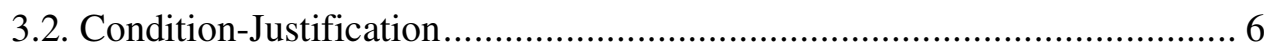

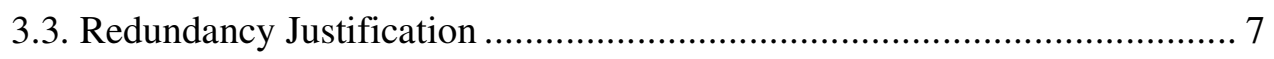

3.4. The Role of These Kinds of Justification.................................................. 8

4. Lakatos and the Importance of Proof-Generated Definitions ........................... 9

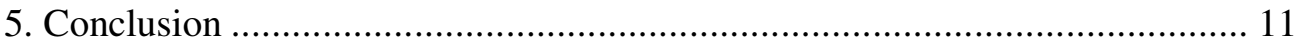

This article has been published in:

M. Suárez, M. Dorato and M. Rédei (eds), EPSA Philosophical Issues in the Sciences: Launch of the European Philosophy of Science Association. Springer.

The final publication is available at:

http://www.springer.com/philosophy/epistemology+and+philosophy+of+science/book/97890-481-3251-5 


\section{Introduction}

The definitions mathematicians work with are not arbitrary, i.e. usually there are good reasons why a definition is regarded as worth considering. This thought motivates the following general question, which will be at the centre of this article: in what ways are definitions justified in the mathematical practice, and are these ways of justifying definitions reasonable? By a justification of a definition I mean the reasons which are given for the definition. In this article we will only consider explicit definitions which introduce a new expression by stipulating that it is equivalent to an already known expression.

Asking about the way a definition is justified means to ask about the kinds of reasons given for these definitions. And reasoning is a core philosophical theme. Consequently, the possible ways of justifying mathematical definitions is an important philosophical theme.

We speak of the formulation of a definition when a mathematician generates a definition she or he has not known before. The initial reason why a mathematician accepts a definition usually makes clear how the formulation of this definition was guided. Hence each kind of justification of definitions gives us a corresponding kind of formulation of definitions. Since the guidance of the formulation of definitions and the justification of definitions are connected in this way, it suffices in what follows to focus only on the justification of definitions.

Apart from Imre Lakatos's work on the justification of definitions, there is very little philosophical discussion on the justification of definitions in the light of actual mathematical practice. As I intend to show in this article, there is much more to say on this issue, and also Lakatos's account of justifying definitions is limited and misguided.

What I will have to say on the above general question will be based on a case study of topological definitions of chaos. In section 2 I will introduce this case study. In section 3 I will then identify the three kinds of justification which are important for topological definitions of chaos. To my knowledge, two of them have not been identified before. After that, in section 4 I will first explain the main theory about the justification of definitions in the light of mathematical practice, namely Lakatos's account of proof-generated definitions. I will then go on to criticise Lakatos's account: as for topological definitions of chaos, in nearly all mathematical fields various kinds of justification are important. 
Some of the theoretical ideas and arguments of this article are developed in more detail in my paper Werndl (2009), where I investigate how notions of randomness in ergodic theory shed light on the justification of definitions.

\section{Case Study: Topological Definitions of Chaos}

Chaotic systems are deterministic systems which are nevertheless highly unstable and show irregular, or even random, behaviour. Due to their instability, chaotic systems exhibit sensitivity to initial conditions (SIC). By SIC we mean the idea that small errors in initial conditions lead to considerably different outcomes.

It was not until the late 1960s that the phenomenon of deterministic yet highly unstable, irregular and sometimes even random behaviour was systematically investigated: catalysed by the development of electronic computers an area of research called 'chaos research' developed (cf. Aubin and Dahan Dalmedico 2002; Dahan Dalmedico 2004). At the end of the twentieth century chaos research boomed. Since then it has been hailed as having led to extraordinarily interesting scientific results and insights, especially in mathematics and physics but also in other scientific disciplines (Ruelle 1991). The Lorenz system, the Hènon system and the logistic map are some of the paradigm chaotic systems.

The theoretical insights of this article on the justification and formulation of mathematical definitions will be based on a case study of topological definitions of chaos, which are discussed in the mathematical field of topological dynamical systems theory. Arguably the main topological definitions of chaos are:

Devaney chaos (three versions), Devaney chaos without periodicity, and the topological entropy (three versions) (cf. Berger 2001, 40; Robinson 1995, 82-83; Smith 1998, chapter 10). ${ }^{1}$

I investigated how these definitions are justified, how the formulation of these definitions was guided, and whether these kinds of justification are reasonable. I chose this case study because the kinds of justification which play a role for topological definitions of chaos

\footnotetext{
${ }^{1}$ There are two main branches of dynamical systems theory which discuss chaotic behaviour, namely topological dynamical systems theory and measure-theoretic dynamical systems theory (ergodic theory). For more on notions of chaos in ergodic theory, see Werndl (2009).
} 
seemed to me widespread in mathematics but different to the ones usually discussed in the philosophy literature.

Before we can turn our attention to the way definitions are justified, I have to introduce the mathematical framework necessary to formulate topological definitions of chaos, namely the concept of a topological dynamical system. To understand the mathematics that follows, it will be enough to have basic knowledge of the theory of metric spaces.

A dynamical system is a mathematical model consisting of a phase space, the set of all possible states of the system, and an evolution equation which describes how solutions evolve in phase space. Dynamical systems often model natural systems.

Definition 1. A topological dynamical system is a triple $(X, d, \mathrm{~T})$, where $X$ is a set the phase space), $d$ is a metric on $X$ and $\mathrm{T}: X \rightarrow X$ is a continuous map (the evolution equation). ${ }^{2}$

The dynamics of the system is given by $x_{n+1}=\mathrm{T}\left(x_{n}\right), x_{0} \mathrm{eX}, \mathrm{n} \in \mathbf{N}_{0}$ and the solution through $x$ is the sequence $\left(\mathrm{T}^{\mathrm{n}}(x)\right)$, where $\mathrm{n} \in \mathbf{N}_{0}{ }^{3}$

With this background we are now ready to look at the kinds of justification which play a role for topological definitions of chaos.

\section{Kinds of Justification}

\section{A. Natural-World-Justification}

First, I assert that topological definitions of chaos are often justified because they capture a preformal idea regarded as valuable for describing or understanding the natural world. I will call such definitions natural-world-justified definitions.

\footnotetext{
${ }^{2}$ To characterise chaotic behaviour, we want to be able to measure the distance between points in phase space. Hence we not only need a topology but also a metric on the phase space.

${ }^{3}$ To be precise, we are discussing here topological dynamical system where time increases in discrete steps. There are also topological dynamical systems where time varies continuously, and they usually derive from differential equations. Definitions of chaos are essentially the same for dynamical systems with discrete and continuous time; hence it suffices to treat the discrete case.
} 
Natural-world-justified definitions are a subgroup of preformal-justified definitions definitions which are justified because they capture a preformal idea regarded as valuable. Several philosophers endorse the general idea that mathematical definitions should capture a valuable preformal idea (cf. Brown 1999, 109].

We of course assume that it is important to describe and understand the natural world. Thus, if the preformal idea is valuable or expected to be valuable for describing and understanding the natural world, natural-world justification is reasonable. Clearly, there can be debates about what makes a preformal idea valuable in this sense.

If a definition is natural-world-justified, or generally preformal-justified, this does not imply that it is a 'best' definition of a vague idea. This may, or may not be the case. For instance, as we will see, several definitions of chaos are natural-world-justified, but there is no 'best' definition among them (cf. Smith 1998, 175).

Many topological definitions of chaos are natural-world-justified, namely a version of Devaney chaos, Devaney chaos without periodicity, the three versions of the topological entropy, and the definition of chaos which says that a system is chaotic if and only if it has a positive topological entropy (Bowen 1978, 17; Petersen 1983, 266-267, Robinson 1995, 8384). Let me now discuss Devaney chaos to illustrate the idea of natural-world-justified definitions.

\section{Devaney Chaos}

Definition 2. $(X, d, \mathrm{~T})$ is Devaney chaotic if and only if

(i) it exhibits sensitive dependence on initial conditions:

$\exists \delta>0 \forall x \in X \forall \mathcal{E}>0 \exists y \in X \exists \mathrm{n} \in \mathbf{N}_{0}\left(\mathrm{~d}(x, y)<\mathcal{E}\right.$ and $\left.d\left(\mathrm{~T}^{\mathrm{n}}(x), \mathrm{T}^{\mathrm{n}}(y)\right)>\delta\right) ;$

(ii) it is transitive:

$\forall U \subseteq X, U \neq \varnothing$ and open, $\forall V \subseteq X, V \neq \varnothing$ and open $\exists \mathrm{n} \geq 0 \mathrm{~T}^{\mathrm{n}}(U) \cap V \neq \varnothing$;

(iii) the set of periodic points of $X$ is dense in $X$.

The standard justification for condition (i) is that it captures SIC: for every initial condition there is another arbitrary close initial condition such that the solutions originating from these initial conditions eventually separate considerably (more than $\delta$ ) (Devaney 1986, 49; Robinson 1995, 82; Smith 1998, 167). As to (ii), Banks et al. (1992, 332) and Berger (2001, 34) follow Devaney in justifying transitivity as corresponding to indecomposability, i.e. that 
the 'system cannot be decomposed into two disjoint open sets which are invariant under the map' (Devaney 1986, 49-50). Transitivity is also justified as capturing the idea of irregularity that any bundle of initial conditions wanders all over the phase space (Smith 1998, 169). The usual justification of condition (iii) is that it captures the idea that in any arbitrary small region of phase space there are periodic points (e.g. Devaney 1986, 50).

Devaney $(1986,50)$ combined these three conditions so as to propose a definition of chaos. In the literature the conjunction of these three conditions is regarded as important because it captures a preformal idea which is valuable for describing or understanding the natural world. Thus Definition 2 is natural-world-justified (Devaney 1986, 49-50; Robinson 1995, 82-84; Smith 1998, 167-170).

As explained above, condition (ii) is sometimes justified as indecomposability. Yet I think that many systems are, beyond doubt, indecomposable but not transitive, e.g. the system defined by iteration of $\mathrm{T}(x)=x^{2}$ on $(0,1)$ is indecomposable but not transitive. ${ }^{4}$ Hence this justification and the corresponding justification of Devaney chaos is problematic. Generally, if the definition does not capture the idea it is said to capture, the justification is problematic because it is unclear why exactly this definition is chosen.

Let me now turn to the second kind of justification I have identified.

\section{B. Condition-Justification}

I claim that another kind of justification plays a role for topological notions of chaos, namely: a definition is justified by the fact that it corresponds to a mathematically valuable condition, i.e. it is equivalent in an allegedly natural way to a previously specified condition which is regarded as mathematically valuable. I will refer to these definitions as condition-justified definitions.

If the previously specified condition is mathematically valuable and the equivalence is mathematically natural, condition-justification is a reasonable kind of justification. To illustrate the idea of condition-justification, let me now discuss a version of Devaney chaos the only topological definition which is condition-justified.

\footnotetext{
${ }^{4}$ It is not transitive because no point in $(0,1 / 2)$ ever enters $(1 / 2,1)$.
} 


\section{Devaney Chaos}

Recall Definition 2 of Devaney chaos. This definition is conjunctive and for reasons of simplicity one might search for a single condition that is equivalent to this conjunctive definition. Guided by this, Touhey (1997) arrives at the following definition; he aims to define chaos for an infinite $\mathrm{X}$, and for this domain his definition is equivalent to Devaney chaos.

Definition 3. The system $(X, d, \mathrm{~T})$, where $\mathrm{X}$ is infinite, is Devaney chaotic if and only if $\forall U \subseteq X, U \neq \varnothing$ and open, $\forall V \subseteq X, V \neq \varnothing$ and open, $\square \exists$ periodic point $p \in U$,

$\exists \mathrm{n} \in \mathbf{N}_{0}$ such that $\mathrm{T}^{\mathrm{n}}(p) \in V$.

Touhey $(1997,411)$ expresses concerns that this definition does not capture a valuable preformal idea of chaos. Smith $(1998,176)$ remarks that the emphasis of this definition on periodicity speaks against Devaney chaos.

Yet I think that Touhey and Smith are misguided here. True, Definition 3 does not capture a preformal idea of chaos, but the question is whether it has to. Let us assume that Devaney's original definition (Definition 2) is accepted as a definition of chaos and that our aim is to define chaos for an infinite phase space. Then Definition 3 can be justified by the fact that it is a single condition being equivalent to Definition 2, regardless of whether it expresses a valuable preformal idea. This makes the definition condition-justified. For other examples of condition-justified definitions, see my paper Werndl (2009).

Generally, condition-justified definitions may in other contexts also capture a valuable preformal idea. However, as for Definition 3 often this won't be the case. Then there is the danger of not appreciating that a definition is condition-justified and asserting instead that this definition captures a meaningful preformal idea, when in fact this is not the case.

Let us now turn to the third kind of justification which plays a role for topological notions of chaos.

\section{C. Redundancy Justification}

I claim that another kind of justification is important for topological notions of chaos, namely that a definition is justified because it eliminates at least one redundant condition in an already accepted definition. Eliminating redundant conditions can be regarded as reasonable. 
Then if the already accepted definition is mathematically valuable, this kind of justification is reasonable. To illustrate the idea of redundancy-justification, let us look at the only topological definition of chaos which is redundancy-justified: again a version of Devaney chaos.

\section{Devaney Chaos}

Recall Definition 2 of Devaney chaos. For an infinite phase space $X$ it can be proven that a transitive systems with dense periodic points exhibits sensitive dependence on initial conditions, i.e. that the conditions (ii) and (iii) imply (i) (Banks et al. 1992). Consequently, many who want to define Devaney chaos for an infinite phase space choose the following definition with the justification that it eliminates a redundant condition (e.g. Banks et al. 1992):

Definition 4. The system $(X, d, \mathrm{~T})$, where $X$ is infinite, is Devaney chaotic if and only if it is transitive and has dense periodic points.

Hence Definition 4 is redundancy-justified.

As we explained for condition-justified definitions in the previous subsection, redundancyjustified definitions may or may not capture a specific valuable preformal idea. If not, there is the danger of not understanding that a definition is redundancy-justified and asserting that it captures a valuable preformal idea, when this is not the case.

\section{D. The Role of These Kinds of Justification}

To conclude, I have identified three kinds of justification which play a role for topological notions of chaos, namely natural-world-justification, condition-justification and redundancyjustification. To the best of my knowledge, condition-justification and redundancyjustification have never been identified before.

Already our above discussion makes clear that these three kinds of justification are different in the sense that there are definitions which are only justified in one way but not in any other way: for instance, as a definition of chaos Definition 2 is only natural-world-justified, Definition 3 is only condition-justified and Definition 4 is only redundancy-justified. 
Furthermore, I claim that each of these three kinds of justification are widespread in mathematics $\}$ and thus are among the most important ones in mathematics.

Let us now see how our insights contribute to the philosophical literature on the justification of mathematical definitions.

\section{Lakatos and the Importance of Proof-Generated Definitions}

Generally, there is little philosophical reflection on the actual practice of how definitions are justified and formed in mathematics. In the relatively recent literature Larvor $(2001,218)$ and Feferman $(1978,321)$ acknowledge the importance of researching the justification and formulation of definitions. Furthermore, as already mentioned, several philosophers argue that definitions in mathematics should be an adequate explication of a preformal idea (cf Brown 1999, 109).

But the main philosopher to have written on the mathematical practice of justifying definitions is still Lakatos (1976 and 1978). Lakatos's contribution here is the concept of a proof-generated definition. His main example for proof-generated definitions are definitions of polyhedron, which are justified because they are needed to make the proof of the Eulerian conjecture go trough, viz. $\backslash$ that for every polyhedron the number of vertices minus the number of edges plus the number of faces equals two:

PI: Proof-generated concepts are neither 'specifications' nor 'generalisations' of naive concepts. The impact of proofs and refutations on naive concepts is much more revolutionary than that: they erase the crucial naive concepts completely and replace them by proof-generated concepts.

The naive term 'polyhedron', even after being stretched by refutationists, denoted something that was crystallike, a solid with 'plane' faces, straight edges. The proof-ideas swallowed this naive concept and fully digested it. In the different proof-generated theorems we have nothing of the naive concepts. That disappeared without trace. Instead each proof yields its characteristic proof-generated concepts, which refer to stretchability, pumpability, photographability, projectability and the like (Lakatos 1976, 89-90, original emphasis). ${ }^{5}$

Unfortunately, Lakatos does not state exactly what proof-generated definitions are (cf. Lakatos 1976, 89-92 and 27-154; Lakatos 1987, 95-97). Obviously, a mathematical

\footnotetext{
${ }^{5}$ It is these properties of stretchability, pumpability, photographability, projectability etc., which eventually define 'polyhedron' and which are needed to make the different versions of the proof of the Eulerian conjecture work.
} 
definition justified in any way is eventually involved in some proofs. If this were not the case, the definition would not be of interest. Therefore, Lakatos cannot have meant that a proofgenerated definition is simply a definition which is eventually involved in proofs.

The following characterisation of a proof-generated definition most plausibly captures what Lakatos means and applies to all his examples: a proof-generated definition is a definition that is needed in order to prove a specific conjecture regarded as valuable (Lakatos 1976, $88-92,127-133,144-154 .^{6}$

Lakatos thinks that in the case of his examples proof-generation is a reasonable way of justifying definitions: with proof-generation the mathematical aim to prove interesting theorems has been reached (Lakatos 1976, 90-92, 128, 148-149, and 153]. Generally, proofjustification is a reasonable way of justifying definitions if the conjecture that should be established is mathematically valuable.

Lakatos also never states clearly how widely he thinks that his account of proof-generated definitions applies. As Leng $(2002,11)$ remarks, his way of writing in his book 'Proofs and Refutations' (1976) suggests that definitions in mathematics should be generally proofgenerated and after the discovery of proof-generation are also proof-generated. However, Lakatos's (1976) book derives from his Ph.D. thesis (Lakatos 1961); and as Larvor (1998) has pointed out, in this thesis Lakatos emphasises that he does not think to have discovered a unique logic of discovery. But given the remarks in his book (Lakatos 1976, 91-92 and 144], he surely must have been convinced that his ideas are representative for several mathematical fields. Therefore, most plausibly, Lakatos held at least that mathematical fields where proofgeneration is, and also should be, the sole important way of how definitions are justified are not exceptional.

\footnotetext{
${ }^{6}$ Lakatos discusses other ways of justifying definitions too: monster-barring, exception-barring, monsteradjustment and monster-including. Like proof-generation these kinds of justification are ways of dealing with counterexamples to conjectures, and Lakatos regards them as inferior to proof-generation (Lakatos 1976, 14-33, 83-87). Moreover, Lakatos (1976, 14-42 and 136-140) claims that they were only employed when the "better" kind of justifying definitions, namely proof-generation, was not yet known. For these reasons and since these other kinds of justification do not play a role for topological definitions of chaos, we won't discuss them further in this article.
} 
Yet, as our case study of topological notions of chaos suggests, this seems wrong. More specifically, I think that, as in our case study, for nearly all mathematical fields after the discovery of proof-generation many different ways of justifying definitions are found. And I also think that for nearly all mathematical subjects various different ways of justifying definitions are reasonable. Actually, I would wonder if a mathematical field could be found where all definitions are, or should be, proof-generated. As I have argued in my paper Werndl (2009), even for the mathematical fields Lakatos (1976) discusses, proof-generation is not the only important form of justification. Thus, to summarise, while Lakatos's ideas are profound, his account is limited and also misguided because he only focused on proofgenerated definitions.

\section{Conclusion}

The general theme of this article has been the actual practice of how definitions are justified in mathematics. The theoretical insights of this article were based on a case study of topological definitions of chaos, and this case study was introduced in section 2. In section 3 I have identified the three kinds of justification which are important for topological definitions of chaos: natural-world-justification, condition-justification and redundancy-justification. To the best of my knowledge, the latter two have not been identified before. I have argued that these three kinds of justification are reasonable and that they are among the most important ones in mathematics. Finally, in section 4 I have discussed the main philosophical theory about the justification of definitions in the light of actual mathematical practice, namely Lakatos account of proof-generated definitions. I have criticised Lakatos's account as being limited and also misguided: as for topological definitions of chaos, in nearly all mathematical fields various kinds of justification are found and are also reasonable.

Acknowledgments. Most thanks should go to Jeremy Butterfield and Peter Smith for their valuable feedback on previous versions of this manuscript. Many thanks also to Roman Frigg, Franz Huber, Brendan Larvor, Mary Leng, Paul Weingartner and the audiences at the 1st London-Paris-Tilburg Workshop in Logic and Philosophy of Science and the 1st Conference of the European Philosophy of Science Association for helpful and interesting discussions and comments. I am grateful to St John's College, Cambridge, for financial support. 


\section{List of References}

Aubin, David, and Amy Dahan-Dalmedico. 2002. Writing the history of dynamical systems and chaos: Longue durèe and revolution, disciplines and cultures. Historia Mathematica 29:273-339.

Banks, John, Jeffrey Brooks, Grant Cairns, Gary Davis, and Peter Stacey. 1992. On Devaney's Definition of chaos. American Mathematical Monthly 99: 332-334.

Berger, Arno. 2001. Chaos and chance: an introduction to stochastic aspects of dynamics. New York: De Gruyter.

Bowen, R. 1978. Topological entropy. In The structure of attractors in dynamical systems Proceedings, North Dakota State University June 20-24, 1977, eds. Nelson G. Markley, Martin John C. and William Perrizo, 17-24. Berlin: Springer.

Brown, James R. 1999. Philosophy of mathematics: an introduction to the world of proofs and pictures. London: Routledge.

Dahan-Dalmedico, A. 2004. Chaos, disorder, and mixing: a new fin-de-siècle image of science? In Growing explanations, historical perspective on the sciences of complexity, ed. Matthew Norton Wise, 67-94. Durham: Duke University Press.

Devaney, Robert. 1986. An introduction to chaotic dynamical systems. New York: AddisonWesley.

Feferman, Solomon. 1978. The logic of mathematical discovery vs. the logical structure of mathematics. PSA: Proceedings of the Biennial Meeting of the Philosophy of Science Association 2: 309-327.

Lakatos, Imre. 1961. Essays in the logic of mathematical discovery. Ph.D. thesis, Cambridge.

Lakatos, Imre. 1976. Proofs and refutations. The logic of mathematical discovery, eds. John Worrall and Elie Zahar. Cambridge: Cambridge University Press.

Lakatos, Imre. 1978. Mathematics, science and epistemology. Philosophical papers volume 2, eds. John Worrall and Gregory Currie. Cambridge: Cambridge University Press. Larvor, Brendan. 1998. Lakatos: an introduction. London and New York: Routledge. Larvor, Brendan. 2001. What is dialectical philosophy of mathematics? Philosophia Mathematica 9: 212-229.

Leng, Mary. 2002. Phenomenology and mathematical practice. Philosophia Mathematica 10: 3-25.

Petersen, Karl. 1983. Ergodic Theory. Cambridg: Cambridge University Press. 
Robinson, Clark. 1995. Dynamical systems: stability, symbol dynamics and chaos. Tokyo: CRC

Press.

Ruelle, David. 1991. Chance and Chaos. Princeton: Princeton University Press.

Smith, Peter. 1998. Explaining Chaos. Cambridge: Cambridge University Press.

Touhey, Pat. 1997. Yet another definition of chaos. American Mathematical Monthly

104: 411-414.

Werndl, Charlotte. 2009. Justifying definitions in mathematics-going beyond Lakatos. Philosophia Mathematica 17: 313-340. 